\title{
Peripheral T cell lymphoma: clinical utility of romidepsin
}

This article was published in the following Dove Press journal:

Blood and Lymphatic Cancer:Targets and Therapy

12 June 2012

Number of times this article has been viewed

\author{
Jasmine Zain \\ Kathryn Sawey \\ NYU Langone Medical Center, \\ New York, USA
}

Correspondence: Jasmine Zain NYU Langone Medical Center, 160 East 34th Street, II th Floor, New York, NY 10016, USA

$\mathrm{Tel}+\mathrm{I} 21273$ I 6544

Fax + I 2I273| 5540

Email jasmine.zain@nyumc.org
Introduction: Direct therapeutic targets, such as aberrant tumor cell genes and tumor cell markers, have been the focus of cancer treatment for more than 50 years. The resulting damage to normal cells and emergence of drug-resistant tumor cells after exposure to conventional chemotherapy have led researchers to study indirect targets, like the tumor vasculature. A more recent indirect approach involves targeting the epigenetic modifiers, DNA methyltransferase and histone deacetylase. Histone deacetylase inhibitors have been shown to be active cytotoxic agents in T cell lymphoma. The current treatments approved by the US Food and Drug Administration for relapsed cutaneous $T$ cell lymphoma are vorinostat and romidepsin. The diversity and rarity of peripheral $\mathrm{T}$ cell lymphomas present a challenge for effective treatment. With their poor overall survival rate, new targeted therapies need to be developed.

Keywords: peripheral T cell lymphoma, treatment, romidepsin

\section{Peripheral T cell lymphomas}

Peripheral T cell lymphoma (PTCL) is classified by the World Health Organization into over 22 subtypes based on clinicopathologic features. ${ }^{1}$ The most common histologies are peripheral $\mathrm{T}$ cell lymphoma not otherwise specified, angioimmunoblastic $\mathrm{T}$ cell lymphoma, and anaplastic large cell lymphoma; the most aggressive are the gamma-delta $\mathrm{T}$ cell lymphomas, including hepatosplenic $\mathrm{T}$ cell lymphoma and cutaneous gammadelta $\mathrm{T}$ cell lymphoma. Adult $\mathrm{T}$ cell lymphoma/leukemia is associated with the human T cell lymphotropic 1 virus, which is prevalent in the Caribbean islands and Far East Asia, explaining some of the geographic variations in the distribution of PTCL around the world. The enteropathy-associated $\mathrm{T}$ cell lymphoma that arises in the intestinal tract has an association with celiac disease, and has a higher prevalence in northern Europe as compared with other parts of the world. ${ }^{2}$ The diversity of each subtype requires specific therapies, but the overall survival remains poor, at less than $30 \% 5$-year survival, ${ }^{3}$ with the exception of cutaneous $\mathrm{T}$ cell lymphoma (CTCL) that tends to have a more indolent course. ${ }^{4}$ Thus, recurrent and refractory disease remains common, and there is a dire need for newer therapies to treat these diseases. The current initial treatment for PTCL is an anthracycline-based regimen, followed by high-dose consolidation chemotherapy and stem cell transplant. ${ }^{3}$ Histone deacetylase (HDAC) inhibitors have shown promising clinical activity in PTCL and are described in further detail in this paper.

\section{HDAC inhibitors}

HDAC inhibitors are epigenetic agents that regulate gene transcription by physical alterations of either DNA or the structural components of chromatin. ${ }^{5}$ 
The opposing activities of histone acetyltransferases and HDACs regulate gene expression by altering chromatin structure. Histone acetyltransferases, by acetylating histones, produce an open chromatin structure, resulting in greater accessibility of regulatory proteins to DNA. By contrast, HDACs catalyze acetyl group removal, lead to a closed chromosomal configuration and transcriptional repression. HDAC inhibition may permit re-expression of proteins that promote apoptosis and cell differentiation while inhibiting cell cycling and cell division. ${ }^{6-7}$ Currently, the 18 HDACs identified in human cells are divided into four classes, based on their homology with HDACs found in yeasts. Class I, II, and IV HDACs require zinc for their enzymatic activity, whereas class III HDACs, also known as silent informant regulator proteins (sirtuins), require nicotinamide adenine dinucleotide $\left(\mathrm{NAD}^{+}\right)$as a coenzyme for their activity. ${ }^{8-10}$

HDAC inhibitors are a chemically broad group of molecules found to inhibit the activity of HDACs at a wide range of concentrations, from low nanomolar to high millimolar levels. To date, there are several HDAC inhibitors in development, with the focus on greater potency and improved tolerability. HDAC inhibitors have shown clinical activity in the treatment of PTCL. ${ }^{7}$ HDAC inhibitors are classified as short-chain fatty acids, hydroxamic acids, benzamides, and cyclic peptides. The bulk of the clinical experience with these compounds has focused on the panHDAC inhibitors (ie, those inhibiting class I, II, and IV enzymes). By inhibiting protein deacetylation and causing accumulation of acetylated histones and nonhistone proteins in the cell, HDAC inhibitors have several effects including transcriptional modification and altered function of proteins regulating cell proliferation, cell cycle progression, differentiation, and apoptosis. ${ }^{5}$ HDAC inhibitors block cell proliferation and cause apoptosis in human tumor-derived cell lines by causing cell cycle arrest in various stages, ie, gap 1 (G1) or gap 2/mitosis (G2/M) phase with relative sparing of normal cells through deregulation of proteins that control cell cycle progression and coordinate the G1/S and G2/M transition, such as cyclins, cyclin-dependent kinases, and their associated regulators. HDAC inhibitors can also cause upregulation of p21, p27, and p16, which bind to and inactivate CDK2 and CDK4, hence leading to inhibition of cell cycle progression. HDAC inhibitors have been observed to increase the expression of genes that encode for death receptors and their ligands, such as Fas and the Apo 2L/TRAIL receptors, DR4 and DR5, downregulate c-FLIP, c1AP2, and $\mathrm{X} 1 \mathrm{AP}$, and induce generation of reactive oxygen species, all of which contribute to apoptosis. Other nonhistone targets of
HDAC inhibitors include transcriptional coregulators, DNA binding transcriptional factors, chaperone proteins, steroid receptors, and DNA repair enzymes. HDAC inhibitors have been shown to have antiangiogenic effects by various mechanisms, including upregulation of angiogenesis inhibitors, such as thrombospondin and von Hippel Lindau factor, as well as downregulation of factors that promote vasculogenesis, such as vascular endothelial growth factor (VEGF) and hypoxia-induced factor-alpha $(H I F-a) \cdot{ }^{5,7,11-13} \mathrm{As}$ single agents, these drugs appear to have a class effect in $\mathrm{T}$ cell lymphoma, in particular CTCL. Two HDAC inhibitors, vorinostat $\left(Z_{\text {Zolinza }}{ }^{\circledR}\right)^{14}$ and romidepsin (FK228, depsipeptide, Istodax $\left.^{\circledR}\right),{ }^{15}$ are approved for the treatment of relapsed or refractory CTCL in the US. Romidepsin is also approved for the treatment of relapsed or refractory PTCL in the US. ${ }^{16}$

\section{Mechanism of action}

Romidepsin was first isolated from the fermentation broth of Chromabacterium violaceum strain WB968 by Fujisawa Pharmaceutical Company in Japan during the course of a program to look for inhibitors of oncogene function. ${ }^{17-18}$ The compound was found to morphologically reverse Ha-ras transformed cells to normal. It was also found to prolong the lives of mice bearing murine ascitic tumors, murine leukemias, and melanoma, and to inhibit the growth of murine solid tumors, such as colon cancer, sarcomas, and human solid tumors, implanted in normal and nude mice. Hence it was isolated as a lipophilic peptide that possesses a characteristic noncysteine disulfide linkage. Subsequently it was noted to be a potent HDAC inhibitor. ${ }^{19,20}$ Furumai et al demonstrated that FK228 is converted to its active reduced form in tumor cells and strongly inhibits HDAC1 and HDAC2 class I enzymes, and is weaker in inhibiting HDAC4 and HDAC6 class II enzymes. ${ }^{1}$ This property makes romidepsin a relatively unique HDAC inhibitor. ${ }^{21}$

HDAC inhibitors work through a myriad of different mechanisms, including upregulation of cell-dependent kinase inhibitors, like p21/p27, and downregulation of cyclin $\mathrm{D}_{1}$, acetylation of nonhistone proteins, including STAT-3, RelA/p65, p53, hypoxia-inducible factor-1 alpha, Bcl-6, and heat shock protein 90 , in a way that modulates their activity and thus their ability to regulate cell growth and survival, as well as direct activation of apoptotic pathways by affecting the balance between antiapoptotic proteins like Bcl-2 and proapoptotic proteins like Bax and Bak. ${ }^{9,10}$ Despite many pleiotropic effects, it has been difficult to assign a precise mechanistic basis to any one or more of these drugs in any particular tumor type, let alone PTCL. It is peculiar 
that these drugs appear to have a class effect in PTCL in contrast with other subtypes of lymphoproliferative neoplasms. Gene expression profiling on paired tissue samples and studies of selected biomarkers including gene activation with HDAC inhibitors has shown that up to $5 \%-10 \%$ of the genome can be affected by HDAC inhibitors. In one study, the genes that were consistently affected included those affecting the cell cycle (CCNDI, IGFI) apoptosis (septin 10, TEF, SORBBS2), angiogenesis (GUCY1A1, ANGPT1), and immune modulation (LAIR1)., ${ }^{52}$

\section{Pharmacokinetics and metabolism}

Romidepsin is extensively metabolized in vivo, primarily by cytochrome P450 (CYP) 3A4, and to a lesser extent by CYP3A5. ${ }^{23}$ A preclinical study in rats showed that $66 \%$ of the dose was excreted into the bile, mediated via the ATP-binding cassette transporter ABCB 1(P-glycoprotein, MDR1), for which romidepsin has been identified as a substrate. ${ }^{24}$ Romidepsin is also likely to be a substrate of the organic transporter, OATP1B3, an influx transporter encoded by SLCO1B3, because other cyclic peptides have been shown to interact with the same transporter. ${ }^{25,26}$ However, the role of single nucleotide polymorphisms in CYP3A4/5, ABCB1, and SLCO1B3 in the individual variability of romidepsin disposition has not been quantitatively assessed. Population pharmacokinetic analysis was performed in 98 patients enrolled in a Phase II multicenter clinical trial of romidepsin in patients with CTCL and PTCL and dosed at $14 \mathrm{mg} / \mathrm{m}^{2}$ or $18 \mathrm{mg} /$ $\mathrm{m}^{2}$ on day 1 during their first treatment cycle. ${ }^{27}$ Population modeling was done using a nonlinear mixed effects approach to explore the effects of polymorphic variations in CYP3A4, CYP3A5, SLCO1B3, and ABCB1, all of which encode genes thought to be involved in the disposition of romidepsin. After a 4-hour infusion, romidepsin was rapidly eliminated from the circulation, with a short half-life of approximately 3.5 hours, as observed in other studies. The disposition of romidepsin followed a polyexponential decline and was similar across doses in the range of $1-24.9 \mathrm{mg} / \mathrm{m}^{2}$. Pharmacokinetics were not affected by repeated dosing. Population clearance was $15.9 \mathrm{~L} /$ hour, with a patient variability of $37 \%$. ABCB1 $2677 \mathrm{G}$ $>\mathrm{T} / \mathrm{A}$ variant alleles trended towards a reduced clearance and lower volume of tissue distribution, but this trend was not statistically significant. Genetic variations in CYP3A5 and SLCO1B3 had no effect on systemic exposure. ${ }^{28,29}$ Thereby, it was concluded that moderate interindividual variability in romidepsin pharmacokinetics existed, but no clinically relevant covariates associated with this variability were observed, at least in this population.
The molecular endpoints of romidepsin treatment were evaluated in 61 patients enrolled in the Phase II trial of romidepsin. ${ }^{24}$ The laboratory correlates included histone $\mathrm{H} 3$ acetylation and $\mathrm{ABCB} 1$ gene expression in peripheral blood mononuclear cells, and ABCB1 gene expression in tumor biopsy samples and fetal Hemoglobin $(\mathrm{HgF})$ levels, all of which were increased following romidepsin treatment. The fold-increase in histone acetylation in peripheral blood mononuclear cells at 24 hours was weakly to moderately correlated with peak plasma concentration ( $\mathrm{C} \max$ ) and area under the curve (AUC), and inversely associated with clearance. These findings support the hypothesis that potent HDAC inhibitors are essential for a critical threshold of drug exposure and durable activity. ${ }^{17}$

\section{Safety profile}

The side effect profile of all HDAC inhibitors is fairly uniform across the diverse chemical classes of agents. ${ }^{30-33}$ The most common side effects include fatigue, nausea, and diarrhea. Transient thrombocytopenia is the most common myelosuppressive effect. During the Phase 1 experience with vorinostat, bone marrow examination of patients at their platelet nadir revealed normocellular marrow with somewhat dysplastic-appearing megakaryocytes that appeared to have impaired platelet budding. ${ }^{34}$ Subsequent studies have shown that HDAC inhibition may repress the GATA-1 gene, an important transcription factor for hematopoiesis, leading to a delay in megakaryocyte maturation and thrombocytopenia. ${ }^{35}$

Electrocardiographic abnormalities, such as QT interval prolongation and ST segment abnormalities, were observed in Beagle dogs treated with a rapid infusion of romidepsin. These abnormalities were also noted in Phase I clinical trials. Hence intensive cardiac monitoring was included in the Phase II trial. ${ }^{36}$ Cardiac studies, such as serial electrocardiograms, cardiac enzymes, multiple gated acquisition scans, echocardiograms, baseline 24-hour Holter analysis, and telemetry monitoring, were performed during the first dose of the first cycle. Although $\mathrm{T}$ wave flattening and ST segment depression was observed in more than half of the electrocardiograms, these were not associated with elevation of cardiac enzymes or altered left ventricular function. They were reversible and short-lived. It was hypothesized that QT interval prolongation could be secondary to administration of a serotonin inhibitor for antiemetic therapy and needs further evaluation.

Three cases of reactivation of latent DNA viruses have been reported in patients enrolled in the Phase II multi-institutional study of romidepsin in T cell lymphoma. ${ }^{37}$ 
Two cases of Epstein Barr virus-associated illness and one case of hepatitis $\mathrm{B}$ virus reactivation was observed. The first patient with Epstein Barr virus reactivation died of multiorgan failure from disseminated Epstein Barr virus infection while the second patient responded well to treatment with single-agent rituximab. The patient with hepatitis B virus reactivation was started on treatment with single-agent entecavir, and had a favorable response. It was hypothesized that these complications were likely related to HDAC inhibitor therapy via transcriptional activation of silenced promoters and induction of immunosuppression via depletion of circulating lymphocytes.

\section{Preclinical activity}

Romidepsin demonstrated potent cytotoxicity against human tumor cell lines and in vivo efficacy against both human tumor xenografts and murine tumors. ${ }^{18,38}$ Romidepsin was found to reverse the morphology of the Ha-ras transformed Ras-1 cells and to inhibit their growth. The antitumor activity of this cyclic peptide was confirmed by the National Cancer Institute and was assigned the number NSC-630176. In vitro, it showed potent antitumor activity against a number of human tumor cell lines, including A549, PC-9 lung adenocarcinoma, PC-1 and PC-10 lung squamous cell carcinoma, LX-1 small cell lung carcinoma, MKN28 and MKN74 stomach adenocarcinoma, MCF-7 and ZR-75-1 mammary adenocarcinoma, and Colo201 and SW480 colon adenocarcinoma at low $\mathrm{ng} / \mathrm{mL}$ concentrations. The half maximal inhibitory concentration $\left(\mathrm{IC}_{50}\right)$ values were found to be $<5 \mathrm{nM}$ for a number of cell lines. The compound demonstrated no cytotoxicity against human fibroblasts, but had weak activity against human HE-9 endothelial cells, suggesting significant selectivity. ${ }^{38}$ In vivo, when given intravenously every 4 days, it inhibited growth of several human tumor xenografts, including MX-1 breast carcinoma, LC-6 lung carcinoma, LU-65 large cell lung carcinoma, and SC-6 stomach adenocarcinoma. ${ }^{39}$ Romidepsin showed a lack of cross-resistance with several commonly used cytotoxic agents, but it was identified as a P-glycoprotein substrate. ${ }^{40}$ In preclinical studies, greater antitumor activity was observed using an intermittent schedule of romidepsin than with daily administration because of greater host tolerance to romidepsin and the ability to administer higher individual doses. In addition, it was observed that short infusions (0.5-4.0 minutes) and prolonged infusions ( $>24$ hours) caused the greatest toxicity, and that infusions of 1-4 hours produced the least toxicity, allowing for the highest individual doses. ${ }^{41}$ No serious toxicities were observed in the preclinical assessment of romidepsin. Cardiac toxicity, including elevations in cardiac enzymes and necrosis, with chronic inflammation or neutrophilic infiltration of cardiac muscle on histopathological examination, was observed in shorter dosing schedules. ${ }^{42}$

\section{Phase I trials}

The National Cancer Institute performed a Phase I clinical trial to determine the maximum tolerated dose of romidepsin, toxicity profile, and to characterize the pharmacokinetic profile of a 4-hour infusion of romidepsin given intermittently on days 1 and 5 every 21 days. $^{43}$ Thirty-seven patients with a variety of primary refractory neoplasms were administered the drug on this schedule, which established the maximum tolerated dose (MTD) as being $17.8 \mathrm{mg} / \mathrm{m}^{2}$. Biologically active serum concentrations were achieved with this dose and one partial response was also observed. During intense ${ }^{44}$ cardiac monitoring, no myocardial damage was noted, although some reversible electrocardiographic changes with ST/T wave flattening were observed. In general, it was well tolerated and major dose-limiting toxicities included constitutional symptoms, diarrhea, dysgeusia, and thrombocytopenia. ${ }^{17} \mathrm{~A}$ dose of $14 \mathrm{mg} / \mathrm{m}^{2}$ over 4 hours on a weekly schedule given for 3 out of 4 weeks was established as the dose to investigate further in the Phase II trials.

Given the promising results of the preclinical studies, patients with PTCL were enrolled in a Phase I trial. Piekarz et $\mathrm{al}^{2}$ reported on four patients with refractory $\mathrm{T}$ cell lymphomas (two patients with Sezary syndrome, one patient with tumor stage CTCL, and one patient with PTCL) who received romidepsin at $12.7 \mathrm{mg} / \mathrm{m}^{2}$ or $17.8 \mathrm{mg} / \mathrm{m}^{2}$ as a 4-hour intravenous infusion on days 1 and 5 of a 21-day cycle. To confirm biological activity, histone acetylation assays were performed on the patients' peripheral mononuclear cells. Both patients with Sézary syndrome had a rapid decrease in the percentage of circulating Sézary cells, along with improvement in skin erythema and edema following treatment with romidepsin. There was rapid clearing of all tumors after six cycles of treatment with romidepsin in the third patient, and the fourth patient with peripheral $\mathrm{T}$ cell lymphoma was declared to be in complete remission after the eighth cycle of romidepsin. At the end of this trial, it was concluded that romidepsin demonstrated significant clinical activity in T cell lymphoma and warranted further investigation in a Phase II trial. 


\section{Phase II trials}

Romidepsin was initially approved in November 2009 for the treatment of CTCL in the relapsed setting based on response data obtained from the pooled analysis of two Phase II trials conducted in parallel and presented to the Food and Drug Administration according to a prior agreement. ${ }^{15}$ In total, there were 135 evaluable patients treated with romidepsin. The outcome of the two trials was comparable, with an objective response rate of $34 \%$ and duration of response of $14.9 \%$, and an objective response rate of $35 \%$ and duration of response of 11.1 months in the gloucester pharmaceutical (GPI) and National Cancer Institute trials, respectively. ${ }^{15}$ Approval of romidepsin for PTCL lagged behind its approval for CTCL. The original National Cancer Institute Phase II trial of romidepsin in $\mathrm{T}$ cell lymphoma was expanded to separate out patients with CTCL and those with PTCL, and then further stratified patients based on the amount of prior therapy they received. Overall, stratification consisted of seven treatment arms with the intention of separating out different cohorts of patients with T cell lymphoma based on histology and prior number of therapies. All patients received drug as an intravenous infusion at a dose of $14 \mathrm{mg} / \mathrm{m}^{2}$ on days 1,8 and 15 every 28 days. The two major cohorts were CTCL and PTCL and the data from these two groups of patients have been analyzed and presented separately. Based on the data generated by the National Cancer Institute, the drug was eventually acquired by Gloucester Pharmaceuticals, and two registration-directed clinical trials were launched, one in CTCL and one in PTCL.

For the PTCL cohort, the National Cancer Institute trial included patients with PTCL who had failed at least one prior systemic therapy and were further stratified into less than two or more than two prior systemic therapies. ${ }^{45}$ There were 48 evaluable patients, and all pathology was reviewed centrally. The patients had received a median of three prior therapies, including 18 patients (38\%) who had received a prior stem cell transplant. The overall response rate was reported as $31 \%$, with four $(8 \%)$ complete responses. Patients who received more than two cycles of therapy had a response rate of $44 \%$. The median duration of response was 9 months, with a median time to progression of 12 months. Bertrand et al reported on the results of the large multicenter Phase II study of romidepsin in PTCL conducted by Gloucester Pharmaceuticals. ${ }^{16}$ A total of 131 patients with histologically confirmed PTCL who had failed or were refractory to more than one systemic therapy and had measurable disease were enrolled. The dosing schedule was the same as in the National Cancer Institute study, ie, weekly dosing of romidepsin at $14 \mathrm{mg} / \mathrm{m}^{2}$ on days 1,8 , and 15 every 28 days. The median age of patients was 59.4 years and they had received a median of two prior systemic therapies. In this study, 21 patients (16\%) had received a prior stem cell transplant. The objective response rate was reported to be 34\%, with 19 (15\%) complete responses and 15 (12\%) partial responses. Median duration of response was not reached, because 16 patients with a complete response had not progressed at the time of analysis. These two studies have clearly established the impressive single-agent activity of romidepsin in PTCL, and presentation of the collective data to the Food and Drug Administration has led to the approval of this agent in the setting of relapsed PTCL where patients have failed at least one line of systemic therapy.

\section{Combination therapy}

By targeting specific molecular pathways, HDAC inhibitors lend themselves to endless combinations with other targeted therapies. Paoluzzi et $\mathrm{al}^{46}$ have demonstrated synergistic cytotoxicity of either romidepsin or belinostat in combination with bortezomib, a proteosome inhibitor, against a panel of mantle cell lymphoma cell lines. This combination was shown to induce mitochondrial membrane depolarization and apoptosis in treated cells. A decrease in cyclin D and BcL-xL was also demonstrated. The combination of an HDAC inhibitor and bortezomib has been evaluated in vitro for synergy against CTCL cell lines as well. The combination has demonstrated upregulation of p21 and p27, and increased expression of phosphorylated $\mathrm{p} 38$, which participates in signaling cascade-controlling cellular responses to cytokines and stress. Decreased expression of vascular endothelial growth factor was also demonstrated after treatment with this combination. Clinical trials are now underway to evaluate the combination of various proteosome inhibitors and HDAC inhibitors for the treatment of lymphoma, and PTCL in particular. DNA methylation is involved in malignancy and is seen upon progression in more than $80 \%$ of all solid tumors. Methylation is one of the main physiological processes to induce silencing of gene expression. The clinical use of demethylating agents like 5-azacitidine and decitabine has been developed in myeloid malignancies without any significant activity noted in lymphoid diseases. However, in vitro data are supportive of the combination of HDAC inhibitors with hypomethylating agents for antilymphoma therapy, as shown by the combination of decitabine and romidepsin. ${ }^{47}$ By lowering the apoptotic threshold, 
HDAC inhibitors lend themselves to combination with chemotherapeutic agents, a strategy that is being explored in solid tumors and other malignancies, and will likely be explored in lymphoproliferative disorders. Several trials are now underway looking at the combination of HDAC inhibitors with cytotoxic agents and other targeted therapies, including bexarotene and pralatrexate.

\section{Conclusion}

Romidepsin is a potent HDAC inhibitor, and has shown remarkable clinical activity against a variety of $\mathrm{T}$ cell lymphomas. While there is a significant amount of data regarding the effects of romidepsin on various cellular functions, it has been difficult to pinpoint the exact mechanistic pathway that is modulated in these diseases. The single-agent activity of romidepsin is impressive, but has not shown curative potential. Combination therapies currently in clinical trials are being designed to improve response rates and to increase the duration of response, in the hope of moving romidepsin to the front-line therapies and into a more curative modality.

\section{Disclosure}

The authors report no conflicts of interest in this work.

\section{References}

1. Jaffe ES, Harris NL, Stein H, et al. Classification of lymphoid neoplasms: the microscope as a tool for disease discovery. Blood, 2008;112(12):4384-4399.

2. Rizvi MA, Evens AM, Tallman MS, et al. T-cell non-Hodgkin lymphoma. Blood. 2006;107(4): 1255-1264.

3. Vose J, Armitage $\mathrm{J}$, and Weisenburger $\mathrm{D}$, et al. International peripheral T-cell and natural killer/T-cell lymphoma study: pathology findings and clinical outcomes. J Clin Oncol. 2008;26(25):4124-4130.

4. Willemze R, Jaffe ES, Burg G, et al. WHO-EORTC classification for cutaneous lymphomas. Blood. 2005;105(10):3768-3785.

5. Bhalla KN. Epigenetic and chromatin modifiers as targeted therapy of hematologic malignancies. J Clin Oncol. 2005;23(17):3971-3993.

6. Bereshchenko OR, Gu W and Dalla-Favera R, et al. Acetylation inactivates the transcriptional repressor BCL6. Nat Genet. 2002;32(4):606-613.

7. Bates SE. Epigenetic therapies reach main street. Clin Cancer Res. 2009;15(12):3917.

8. Davie JR. Covalent modifications of histones: expression from chromatin templates. Curr Opin Genet Dev. 1998;8(2):173-178.

9. Cotto M, Cabanillas F, Tirado M, et al. Epigenetic therapy of lymphoma using histone deacetylase inhibitors. Clin Transl Oncol. 2010;12(6):401-409.

10. Bolden JE, Peart MJ, and Johnstone RW. Anticancer activities of histone deacetylase inhibitors. Nat Rev Drug Discov. 2006;5(9):769-784.

11. Batty N, Malouf GG, Issa JP. Histone deacetylase inhibitors as antineoplastic agents. Cancer Lett. 2009;280(2):192-200.

12. Ashkenazi A. Targeting death and decoy receptors of the tumournecrosis factor superfamily. Nat Rev Cancer. 2002;2(6):420-430.

13. Chen Z, Clark S, Birkeland M, et al. Induction and superinduction of growth arrest and DNA damage gene 45 (GADD45) alpha and beta messenger RNAs by histone deacetylase inhibitors trichostatin A (TSA) and butyrate in SW620 human colon carcinoma cells. Cancer Lett. 2002;188(1-2):127-40.
14. Olsen EA, Kim YH, Kuzel TM, et al. Phase IIb multicenter trial of vorinostat in patients with persistent, progressive, or treatment refractory cutaneous T-cell lymphoma. J Clin Oncol. 2007;25(21):3109-3115.

15. Demierre M. Pooled analysis of two international multicenter clinical studies of romidepsin in 167 patients wiht cutaneous lymphoma. 45th American Society of Clinical Oncology Annual Meeting, 2009.

16. Coiffier B, Pro B,Prince HM, et al. Results From a Pivotal, Open-Label, Phase II Study of Romidepsin in Relapsed or Refractory Peripheral T-Cell Lymphoma After Prior Systemic Therapy. J Clin Oncol. 2012;30(6):631-636. Epub 2012 Jan 23.

17. Piekarz RL,Robey R, Sandor V, et al. Inhibitor of histone deacetylation, depsipeptide (FR901228), in the treatment of peripheral and cutaneous T-cell lymphoma: a case report. Blood. 2001;98(9):2865-2868.

18. Ueda H, Manda T, Matusmoto S, et al. FR901228, a novel antitumor bicyclic depsipeptide produced by Chromobacterium violaceum No. 968. III. Antitumor activities on experimental tumors in mice. J Antibiot (Tokyo), 1994;47(3):315-323.

19. Nakajima H, Kim YB, Terano H, et al. FR901228, a potent antitumor antibiotic, is a novel histone deacetylase inhibitor. Exp Cell Res. 1998;241(1):126-133.

20. Ueda H, Nakajima H, Hori Y, et al. FR901228, a novel antitumor bicyclic depsipeptide produced by Chromobacterium violaceum No 968 . I. Taxonomy, fermentation, isolation, physico-chemical and biological properties, and antitumor activity. J Antibiot (Tokyo). 1994;47(3): $301-310$.

21. Furumai R, Matsuyama A, Kobashi N, et al. FK228 (depsipeptide) as a natural prodrug that inhibits class I histone deacetylases. Cancer Res. 2002;62(17):4916-4921.

22. Piekarz RL, Bates SE. Epigenetic modifiers: basic understanding and clinical development. Clin Cancer Res. 2009;15(12):3918-3926.

23. Fouladi M, Furman WL, Chin T, et al. Children's Oncology Group. Phase I study of depsipeptide in pediatric patients with refractory solid tumors: a Children's Oncology Group report. J Clin Oncol. 2006; 24(22):3678-3685.

24. Fouladi M. Histone deacetylase inhibitors in cancer therapy. Cancer Invest. 2006;24(5):521-527.

25. Byrd JC, Marcucci G, Parthun MR, et al. A Phase 1 and pharmacodynamic study of depsipeptide (FK228) in chronic lymphocytic leukemia and acute myeloid leukemia. Blood. 2005;105(3):959-967.

26. Bates SE, Zhan Z, Steadman K, et al. Laboratory correlates for a Phase II trial of romidepsin in cutaneous and peripheral T-cell lymphoma. Br J Haematol. 2010;148(2):256-267.

27. Woo S, Gardner ER, Chen X, et al. Population pharmacokinetics of romidepsin in patients with cutaneous T-cell lymphoma and relapsed peripheral T-cell lymphoma. Clin Cancer Res. 2009;15(4): $1496-1503$.

28. Shiraga T, Tozuka Z, Ishimura R, et al. Identification of cytochrome P450 enzymes involved in the metabolism of FK228, a potent histone deacetylase inhibitor, in human liver microsomes. Biol Pharm Bull. 2005;28(1):124-129.

29. Fehrenbach T, Cui Y, Faulstich H, et al. Characterization of the transport of the bicyclic peptide phalloidin by human hepatic transport proteins. Naunyn Schmiedebergs Arch Pharmacol. 2003;368(5): 415-420.

30. Mann BS, Johnson JR, He K, et al. Vorinostat for treatment of cutaneous manifestations of advanced primary cutaneous T-cell lymphoma. Clin Cancer Res. 2007;13(8):2318-2322.

31. Prince HM, Bishton MJ, Harrison SJ. Clinical studies of histone deacetylase inhibitors. Clin Cancer Res. 2009;15(12):3958-3969.

32. Lane AA, Chabner BA. Histone deacetylase inhibitors in cancer therapy. J Clin Oncol. 2009;27(32):5459-5468.

33. Lyseng-Williamson KA, Yang LP. Romidepsin: a guide to its clinical use in cutaneous T-cell lymphoma. Am J Clin Dermatol. 2012;13(1):67-71.

34. O'Connor OA, Heaney ML, Schwartz L, et al. Clinical experience with intravenous and oral formulations of the novel histone deacetylase inhibitor suberoylanilide hydroxamic acid in patients with advanced hematologic malignancies. J Clin Oncol. 2006;24(1):166-173. 
35. Matsuoka H, Unami A, Fujimura T, et al. Mechanisms of HDAC inhibitor-induced thrombocytopenia. Eur J Pharmacol. 2007;571(2-3): 88-96.

36. Piekarz RL, Frye AR, Wright JJ, et al. Cardiac studies in patients treated with depsipeptide, FK228, in a Phase II trial for T-cell lymphoma. Clin Cancer Res. 2006;12(12):3762-3773.

37. Ritchie D, Piekarz RL, Blombery P, et al. Reactivation of DNA viruses in association with histone deacetylase inhibitor therapy: a case series report. Haematologica. 2009;94(11):1618-1622.

38. Ueda H, Nakajima H, Hori Y, et al. Action of FR901228, a novel antitumor bicyclic depsipeptide produced by Chromobacterium violaceum No 968, on Ha-ras transformed NIH3T3 cells. Biosci Biotechnol Biochem. 1994;58(9):1579-1583.

39. Khan SB, Maududi T, Barton K, et al. Analysis of histone deacetylase inhibitor, depsipeptide (FR901228), effect on multiple myeloma. Br J Haematol. 2004;125(2):156-161.

40. Xiao JJ, Foraker AB, Swaan PW, et al. Efflux of depsipeptide FK228 (FR901228, NSC-630176) is mediated by P-glycoprotein and multidrug resistance-associated protein 1. J Pharmacol Exp Ther. 2005;313(1): 268-276.

41. Page JG, Rodman LE, Heath JE, et al. Effect of infusion rate on toxicity of depsipeptide (NCS-63-176) in Beagle dogs. Proc Am Assoc Cancer Res. 1995;36;368:(Abstr 2193).
42. Li Z, Chan KK. A subnanogram API LC/MS/MS quantitation method for depsipeptide FR901228 and its preclinical pharmacokinetics. J Pharm Biomed Anal. 2000;22(1):33-44.

43. Marshall JL, Rizvi N, Kauh J, et al. A Phase I trial of depsipeptide (FR901228) in patients with advanced cancer. J Exp Ther Oncol. 2002; 2(6):325-332.

44. Piekarz RL, Frye R, Turner M, et al. Phase II multi-institutional trial of the histone deacetylase inhibitor romidepsin as monotherapy for patients with cutaneous T-cell lymphoma. J Clin Oncol. 2009;27(32): 5410-5417.

45. Piekarz RL, Frye R, Prince HM, et al. Phase 2 trial of romidepsin in patients with peripheral T-cell lymphoma. Blood. 2011;117(22):5827-5834.

46. Paoluzzi L, Scotto L, Marchi E, Zain J, Seshan VE, O’Connor OA. Romidepsin and belinostat synergize the antineoplastic effect of bortezomib in mantle cell lymphoma. Clin Cancer Res. 2010;16(2): 554-565.

47. Marchi E, Kalac M, Bongero D, et al. Combination of Epigenetic Agents Synergistically Reverse the Malignant Phenotype in Models of T-Cell Lymphoma ASH Annual Meeting Abstracts 2011;118: 2727.
Blood and Lymphatic Cancer: Targets and Therapy

\section{Publish your work in this journal}

Blood and Lymphatic Cancer: Targets and Therapy is an international, peer-reviewed, open access journal focusing on blood and lymphatic cancer research, identification of therapeutic targets and the optimal use of preventative and integrated treatment interventions to achieve improved outcomes, enhanced survival and quality of life for the

\section{Dovepress}

cancer patient. The manuscript management system is completely online and includes a very quick and fair peer-review system. Visit http://www.dovepress.com/testimonials.php to read real quotes from published authors. 